\title{
Welfare ANalysis of The Number and Locations of Local Public Facilities
}

\author{
by
}

Marcus Berliant, Shin-kun Peng, and Ping Wang

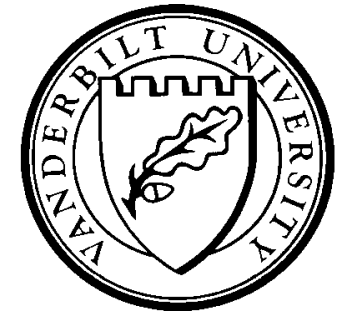

Working Paper No. 00-W35

August 2000

\section{DEPARTMENT OF ECONOMICS \\ VANDERBILT UNIVERSITY \\ NASHVILLE, TN 37235}

www.vanderbilt.edu/econ 


\title{
Welfare Analysis of the Number and Locations of Local Public Facilities
}

\author{
Marcus Berliant \\ Washington University, St. Louis, MO, USA \\ Shin-kun Peng \\ Academia Sinica, Taipei, Taiwan, ROC \\ Ping Wang \\ Vanderbilt University, Nashville, TN, USA \\ This Version: August 2000
}

\begin{abstract}
We develop a discrete or finite household model with congestable local public goods where the level of provision, the number of facilities and their locations are all endogenously determined in a purely normative context. We prove the existence of an equal-treatment identical-provision second best optimum, where all households are required to reach the same utility level, the provision of local public good is required to be the same at all facilities, and all facilities must serve the same number of consumers. Such an optimal public facility configuration need not be geographically centralized even if there is only a single public facility site. Moreover, the optimal public facility configuration could be either concentrated (single site) or dispersed (multiple sites), depending crucially on the degree of congestability and the household valuation of local public goods as well as the unit transportation cost.
\end{abstract}

\section{JEL Classification Numbers: D61, H41, R13}

Keywords: Congestable Local Public Goods, Optimal Public Facility Configurations

Acknowledgments: We are grateful for valuable comments and suggestions from Hideo Konishi, Tomoya Mori, David Wildasin, and participants at the North American Meetings of the Regional Science Association International in Montreal. The first author acknowledges financial support from NSF grant No. SBR9523940. The second author acknowledges support from NSC grant No. 89-2415-H-001-026 and Fulbright Foundation grant No. 21994, and also appreciates the hospitality of the Department of Economics, Washington University at St. Louis during the academic year 1997-1998. We acknowledge financial support from the Institute of Economics of Academia Sinica, which facilitated international collaboration between the authors.

Correspondence: Marcus Berliant, Department of Economics, Washington University, St. Louis, MO 63130-4899, Tel. 314-935-8486, Fax: 314-935-4156, E-mail: berliant@wueconc.wustl.edu; Shin-Kun Peng, Institute of Economics, Academia Sinica, Taipei, Taiwan, Tel:886-2-2782-2791(ext.209), Fax: 886-22785-3946, E-mail: speng@ieas.econ.sinica.edu.tw; Ping Wang, Department of Economics, Vanderbilt University, 415 Calhoun Hall, Nashville, TN 37235, tel:(615)322-2871, fax:(615)343-8495, e-mail: ping.wang@vanderbilt.edu. 


\section{Introduction}

In the literature on local public goods, there are many papers examining the effects of local public goods on the underlying spatial structures. These studies focus primarily on how the level and locations of public goods provision influence the locational choices of households and firms. ${ }^{1}$ The presence of public goods serves as an agglomerative force, leading to the clustering of households and firms around a public facility. While this positive analysis is interesting, it is equally important to examine public facility configuration from a normative point of view. The number and locations of public facilities are crucial in determining the level of economic welfare in a community. However, due to technical barriers, this important task has not been explored completely. In this paper, we attempt such an endeavor by performing a general-equilibrium welfare analysis using a discrete (or finite) household model with congestable local public goods in which both the number of facilities and their locations are endogenously determined. ${ }^{2}$

In contrast with most previous studies in the area of public goods and spatial economics, where models with a continuum households are used, our paper employs a discrete household framework. This is because in continuum models, both the first and the second welfare theorems may fail even without public goods (see Berliant, Papageorgiou and Wang 1990). More importantly, due to a problem associated with a set of zero measure under the continuum setup, an optimum may not exist (see Berliant and Wang 1993). Thus, we prefer to use the discrete framework in order to circumvent these difficulties. Our model structure can be regarded as an extension of the discrete model of Berliant and Fujita (1992) to incorporate locally congestable public goods with the level of provision, the number and locations of the facility sites endogenously determined.

${ }^{1}$ See, for example, Arnott and Stiglitz (1979), Brueckner (1979), Ellickson (1979), Scotchmer (1986), ReVelle (1987), Wildasin (1987) and Thisse and Wildasin (1992).

${ }^{2}$ The discrete model is the original urban model of Alonso (1964). He proposes models with land prices that are additive across parcels, where no arbitrage is possible in equilibrium, as well as models with land prices that are not additive across parcels, such as pricing according to the value of the point in a parcel closest to the city center, where arbitrage is possible in equilibrium. 
The second special feature of our paper is the consideration of locally congestable public goods. Most previous studies focus on the non-exclusive nature of local public goods (e.g., see Fujita 1986, Sakashita 1987, Thisse and Wildasin 1992, Peng 1996 and Hochman and Pines 1997), ignoring the possibility that public goods services may face local congestion depending on the locationally dependent number of users. While Hochman (1982) considers local congestability, the public facility location in his paper is exogenously fixed. Local congestability is certainly observed in reality, as in the case of parks, public schools and libraries, where aggregate usage would affect the quality of services. Theoretically, it is also interesting to investigate how the presence of local congestability may affect the welfare properties of an economy in the spatial context.

The third feature of our paper is that we determine both the number and locations on public facilities and the level of public good provision endogenously. As in the literature, we restrict our focus to "identical provision" of the local public good with the same level of the local public good provided in each site and used by the same number of people. While Fujita (1986), Sakashita (1987) and Hochman and Pines (1997) endogenize the locations of public facilities, the number of public facilities is exogenously given as one in Fujita and as one or two in Sakashita as well as Hochman and Pines. Although Hochman and Pines make utility comparisons between the single and dual public facility cases, there is no endogenous mechanism pinning down the number of public facilities within the system. One may wonder whether the provision of a large central park/library or a few small community parks/libraries is more efficient in the spatial economy. Furthermore, the existing literature on public facilities in the spatial context assumes that the level of provision is exogenously fixed. In contrast, we endogenize the optimal allocation between the public and the private goods but establish a sufficient condition such that the provision of the local public good is independent of the configuration of public facilities and the allocation of the private good.

Finally, in determining the optimal public facility configuration, our paper is the only one, to our knowledge, based on the concept of Pareto optimality. In particular, we construct a concept of "equal- 
treatment" identical-provision optimality that has all households achieving the same level of utility. ${ }^{3}$ Fujita (1986) and Sakashita (1987) employ representative consumer utility maximization, which may be reinterpreted as an equally weighted social welfare maximum of households with concave utility functions. When land rents exit the system (as in Sakashita, for example), the command optimality outcomes need not be consistent with Pareto optimality. In comparing representative utility levels, Hochman and Pines (1997) consider an open model with an endogenous population of households. In their economy, the welfare of people outside the city is ignored and hence Pareto optimality is not well-defined. While our equal-treatment identical-provision optimality concept is well-defined in the general equilibrium sense, the feature of equal treatment allows us to compare our results with the contributions of other studies.

The main findings of this paper are as follows. First, under proper assumptions, we prove the existence of an equal-treatment identical-provision optimum and establish a sufficient condition for the level of public good provision to be independent of the public facility configuration and other endogenous variables. Second, given a single public facility, the equal-treatment optimal location of the public facility may not be at the geographic center and such an optimal location need not be unique. Third, with the number of public facilities endogenously determined, a sufficiently high degree of both congestability and unit transportation cost and a sufficiently low household valuation of the public good imply that the equaltreatment identical-provision optimal public facility configuration is always dispersed. Finally, we show the robustness of our main findings to the number of households and the possibility that an equal-treatment identical-provision optimum may be generally Pareto inferior to a non-identical provision feasible allocation.

In addition to constructing a well-defined and more complete normative analysis of the configuration of facilities providing congestable local public goods, our results contrast with those in the existing literature in several important dimensions. First, the possibility of a non-centralized public facility site and the

${ }^{3}$ However, we will show at the end of Section 2 that with identical provision of local public goods, our concept of optimality generally forces a second best allocation since non-identical provision of local public goods may be Pareto dominant. 
possibility of multiple optimal public facility configurations contrast with all previous studies. Second, when there are two dispersed facility sites, the optimal locations need not be at the first and third quarter of the linear city, contrasting with Sakashita (1987). These discrepancies are mainly due to the difference between the finite versus continuum of households setups and the concept of equal-treatment identical-provision optimality versus command optimality; command optimality is commonly used in the balance of the literature. Third, our conditions for generating dispersed public facility configurations contrast with those in Hochman and Pines (1997) where the unit transportation cost is required to be extremely high or low in order for a dispersed (two-location) configuration to emerge. In particular, the dispersed configuration becomes the unique outcome only when the unit transportation cost is sufficiently large.

In Section 2, we develop a simple spatial model of locally congestable public goods. Section 3 proves the existence of an equal-treatment identical-provision optimum. In section 4 , we characterize optimal public facility configurations with two or more households, using a utility function allowing for the optimal level of public good provision to be independent of public facility configuration and private good allocation. Section 5 examines the case of a non-identical provision of the local public good. Section 6 concludes the paper and provides avenues for future research.

\section{The Economy}

Consider a community accommodating $N$ identical households that must locate in a location $i \in \mathrm{X} \equiv$ $\{1,2,3, \ldots . . N\}$, where $N<\infty$. Each residential region has a unit length in a one-dimensional interval $\mathrm{L} \equiv[0$, $N]$, and is of homogeneous quality (except for the aspect of public goods service). Each household is required to reside in a location. In the absence of vacant land or multiple occupancy, land allocation in our spatial economy becomes a simple assignment problem in which each lot is occupied by only one household. ${ }^{4}$ Thus, household $i$ consumes the interval $x_{i} \equiv(i-1, i]$ and is conveniently labeled by the "front-door" location

${ }^{4}$ This is similar to the Koopmans and Beckmann (1957) linear assignment problem, meaning that it is infeasible to have more than one household residing in the same lot. 
of its residence. The aggregate endowment of composite good is $E>0$. Households are homogenous with respect to their taste (preferences). They consume composite good and travel to one of the specific public facility locations to enjoy the local public good service provided by the community. ${ }^{5}$

The technology used by the community government to produce the single public good is constant returns to scale, generating one unit of public good for each unit of composite good used as an input. ${ }^{6}$ Thus, we let $K$ denote both the composite consumption good used in production as well as the total supply of public good. This total supply is divided among the $\mathrm{n}$ facilities. The public good may be provided at one (concentrated) or more than one (dispersed) location. Denote the set of public facility locations as $\mathrm{H} \subset \mathrm{L}$. When there is a single public facility site at $\eta \in L$, we have $H \equiv\{\eta\}$. All households patronize this facility. When there are $n$ sites, we have $H \equiv\left\{\eta_{1}, \eta_{2}, \ldots, \eta_{n}\right\}$ where $\eta_{j} \in L$ with a corresponding level of the local public good denoted by $K_{j}$, and where $M_{j}$ is the number of households patronizing site $\mathrm{j}(\mathrm{j}=1, \ldots, \mathrm{n})$ (obviously, $\sum_{j=1}^{n} K_{j}=K$ and $\sum_{j=1}^{n} M_{j}=N$ ). Thus, by denoting the cardinality of a discrete set as $\mu$, we have $n=\mu(H)$ and the pair $(n, H)$ summarizes the configuration of public facilities, i.e., both the number and locations of local public facility sites. Examples of such local public goods include schools, libraries, museums, parks, theater halls, public swimming pools, exercise fields or other recreational facilities.

Each household must incur a transportation cost to travel to the nearest public facility. ${ }^{7}$ For

${ }^{5}$ For notational simplicity, we follow the conventional wisdom assuming that each location contains only one resident. More realistically, one may imagine that each residential region $x_{i}$ is occupied by a finite number of identical households. This generalization only requires minor modification of the definition of land partition without changing any of our main results.

${ }^{6}$ In practice, in most countries there are higher level authorities (city, state, region, federal). The higher tier may seek to influence or constrain the activities of local communities by direct methods (local authorities may be forbidden from enacting certain restrictive legislation or compelled to provide a minimum standard of public goods), or by taxation and subsidies (e.g., a matching grant). Also, it is possible that the cost of local public good provision may vary across locations, especially if it requires land as an input and if land is heterogeneous or the population distribution is not uniform. Nevertheless, these issues are far beyond the scope of the present paper.

${ }^{7}$ Hochman (1982) defined a concentrated local public good as a public good that is available to consumers only in specific locations to which households must travel to make use of the service. Fujita 
simplicity, it is assumed that the amount of land required by the facility is negligible. ${ }^{8}$ Moreover, we consider throughout the paper identical provision (henceforth called IP) of the local public good in the sense that when there are multiple public facility sites, the same level of local public good is provided at each site and the same number of households use each of them. Thus, when there are $\mathrm{n}$ sites, the identical provision of the public good implies that each site is equipped with a local public good of size $K_{j}=K / \mathrm{n}$ and shared by a population of households of $M_{j}=N / \mathrm{n}$ (for all $\mathrm{j}=1, \ldots, \mathrm{n}$ ). While the feature of identical provision allows us to compare our results with those in these previous studies, we would like to alert the reader that in general, a non-identical provision of the public good may be Pareto dominant (see Section 5 for a detailed illustration).

At a given location, a household has the nonnegative orthant of $\mathbb{R}^{3}, \mathbb{R}^{3}$, as his consumption set. There are three types of goods: (i) a composite consumption good, (ii) a local public good, whose facility service is valued by each household, and (iii) land. To simplify the analysis, we assume that the demand for land is perfectly inelastic - i.e., each household consumes a fixed, unit quantity of land. Thus, the consumption set reduces to $\mathbb{R}^{2}$.

Let $Y_{i} \subset \mathrm{H}$ be a subset of public facilities to which household $i$ travels and denote $y_{i} \in Y_{i}$ as a travel destination of household $i$. The pecuniary cost of transportation, $\mathrm{T}_{\mathrm{i}}\left(x_{i}, y_{i}\right)$, varies with distance from the public facility location. Since any single point has zero measure, we do not have to differentiate between open and closed intervals and can utilize the inner-Hausdorff metric, denoted $d$, to measure the distance between a single point and a half open interval. ${ }^{9}$ Specifically, the distance between household $i$ 's residential region $x_{i} \in \mathrm{L}$ and a public facility location $y_{i}$, denoted $d\left(x_{i}, y_{i}\right)$, is defined as: (i) $\left(y_{\mathrm{i}}-i\right)$, if $y_{i}>i$ [rightward

(1986) denoted this as a traveled-for public good. Thisse and Wildasin (1992) and Hochman and Pines (1997) employ the same concept in their definition of public goods.

${ }^{8}$ Fujita (1986), Sakashita (1987), Thisse and Wildasin (1992), Peng (1996) and Hochman and Pines (1997) make a similar assumption.

${ }^{9}$ Importantly, the standard Euclidean metric is not applicable in this case. For further discussion, the reader is referred to Klein and Thompson (1984, p. 39) and Berliant and Wang (1993, pp. 130-131.) 
traveling pattern]; (ii) 0 , if $i-1 \leq y_{\mathrm{i}} \leq i$ [non-traveling]; (iii) $(i-1)-y_{i}$, if $y_{i}<i-1$ [leftward traveling pattern], respectively.

In this spatial economy, we assume that the transportation (or commuting) cost schedule (measured in terms of the composite good), T, satisfies:

(A1) (Transport cost) $\mathrm{T}_{\mathrm{i}}=\mathrm{T}\left(d\left(x_{i}, y_{i}\right)\right)$ such that $\mathrm{T}(0)=0, \mathrm{~T}^{\prime}>0$ and $\mathrm{T}^{\prime \prime} \geq 0$.

For example, a linear transport cost schedule can be expressed as:

$$
T_{i}=t d\left(x_{i}, y_{i}\right), t>0
$$

While we impose only (A1) in obtaining general theorems, the simple linear transport cost schedule is used in Sections 4 and 5 below for a complete characterization of optimal public facility configurations. It is clear that with an identical provision of the public good, household $i$ must travel to the closest public facility site $y_{i} \equiv \operatorname{argmin}_{\eta_{j} \in H} d\left(x_{i}, \eta_{j}\right)$. Should this argmin be two points, household $i$ randomizes, with equal probability, traveling to each of the two closest sites. ${ }^{10}$ This randomization assumption is made to be consistent with the consideration of identical public good provision. ${ }^{11}$ Obviously, $\mathrm{n}$ is at most $N$ under this setup and hence $n \in X$.

Let the quantity of composite good consumption and of public good consumption be given by $z$ and $G$, respectively. Let $\alpha$ denote the degree of congestability of the public good. The amount of public service

${ }^{10}$ To be more concrete, consider the case of $\mathrm{N}=3$ and $\mathrm{n}=2$ where the two facility sites are at $\eta_{1}=$ 1 and $\eta_{2}=2$. Thus, a possible travel pattern is: households 1 and 3 commute to $y_{1}=\eta_{1}$ and $y_{3}=\eta_{2}$, respectively, whereas household 2 randomizes by commuting to $y_{2} \in Y_{i}=\left\{\eta_{1}, \eta_{2}\right\}$.

${ }^{11}$ An alternative is to follow Lucas (1990), assuming there is a continuum of household members in each household and the household head makes a joint decision for all members. In this case, we simply assume that half of the members of household $\mathrm{i}$ travel to one site and the remaining half to the other. 
embodying congestion can therefore is specified as: ${ }^{12}$

(A2) (Consumption of public good) $G\left(K_{j}, M_{j}\right)=K_{j}\left(M_{j}\right)^{-\alpha}, \alpha \in(0,1)$.

Under identical provision, recall that the local public good allocated to each site is $K_{j}=K / \mathrm{n}$ and each site is patronized by $M_{j}=N / n$ households. We can thus rewrite: $G=(K / n) /(N / n)^{\alpha}$. Notably, $\alpha=0$ implies a pure public good, whereas $\alpha=1$ implies a private good. For example, if all households are going to share only one concentrated local public good at a single facility of size $K$, then $G=K(N)^{-\alpha}$ which captures the case considered by Hochman (1982).

(A3) (Well-behaved preferences of households) $u(z, G)$ is a strictly increasing in both arguments, strictly concave, and twice continuously differentiable.

An allocation is defined as a list of (i) nonnegative quantities of composite good $z_{\mathrm{i}} \in \mathbb{R}_{+}$consumed by each household $\mathrm{i}$ and indexes $x_{\mathrm{i}} \in \mathrm{X}$ describing each household i's partition of land $(\mathrm{i}=1, \ldots, N)$, (ii) a nonnegative provision of the local public good $K_{j} \in \mathbb{R}_{+}$at each location $\eta_{j} \in \mathrm{L}\left(\mathrm{j}=1, \ldots, \mathrm{n}\right.$ and $\left.\sum_{j=1}^{n} K_{j}=K\right)$, the number of households patronizing to each public facility site $M_{j} \in \mathbb{R}_{+}\left(\mathrm{j}=1, \ldots, \mathrm{n}\right.$ and $\left.\sum_{j=1}^{n} M_{j}=N\right)$, together with a configuration of public facilities indicated by $(n, H)$ where $n=\mu(H) \in X$ and $H \equiv\left\{\eta_{1}, \eta_{2}, \ldots\right.$, $\left.\eta_{\mathrm{n}}\right\} \subset \mathrm{L}$, and (iii) a subset of location indexes $Y_{i} \equiv\left\{y_{\mathrm{i}}\right\} \subset \mathrm{H}(\mathrm{i}=1, \ldots, N)$ containing each public facility site $y_{i}$ to which a particular household $i$ patronize. In short, an allocation can be written as: $a=\left(\left\{z_{\mathrm{i}}, Y_{\mathrm{i}}\right\}_{\mathrm{i}=1, \ldots, \mathrm{N}},\left\{K_{\mathrm{j}}\right.\right.$ ,$\left.\left.M_{\mathrm{j}}\right\}_{\mathrm{j}=1, \ldots, \mathrm{n}}, \mathrm{n}, \mathrm{H}\right)$.

Given $y_{i} \in Y_{i} \subset \mathrm{H}(\mathrm{i}=1, \ldots, N)$, the material balance condition for the composite good is:

${ }^{12} \mathrm{~A}$ local public good subject to the external effect of congestion is defined as a congestable local public good. For detailed discussion of congestable public goods, see Hochman (1982) and Starrett (1991). 


$$
\sum_{i=1}^{N}\left[z_{i}+T\left(d\left(x_{i}, y_{i}\right)\right)\right]=E-K .
$$

We can now define a feasible allocation as an allocation $a=\left(\left\{z_{\mathrm{i}}, Y_{\mathrm{i}}\right\}_{\mathrm{i}=1, \ldots, \mathrm{N}},\left\{K_{\mathrm{j}}, M_{\mathrm{j}}\right\}_{\mathrm{j}=1, \ldots, \mathrm{n}}, \mathrm{n}, \mathrm{H}\right)$ satisfying the material balance condition (2).

We next restrict our attention to the case of identical provision of the local public good. An identical-provision feasible allocation is a feasible allocation such that $K_{\mathrm{j}}=K / \mathrm{n}$ and $M_{\mathrm{j}}=\mathrm{N} / \mathrm{n}$ for all $\mathrm{j}=1$, $\ldots, n$. An identical-provision optimum is an IP feasible allocation such that there is no other IP feasible allocation making no household worse-off and at least one better-off. Under assumption (A2) together with household randomization and identical provision, public good service becomes:

$$
G(n, K)=K /\left(N^{\alpha} n^{1-\alpha}\right),
$$

which is constant across all households. The term $N^{\alpha} n^{1-\alpha}$ can be regarded as a "net congestion factor," increasing in the population and the number of public facility sites. Since land partition in our spatial economy is trivial and identical local public good provision enables us to write $K_{\mathrm{j}}=K / \mathrm{n}$ and $M_{\mathrm{j}}=\mathrm{N} / \mathrm{n}$ for all $\mathrm{j}$, the IP optimality problem is simply given by:

$$
\begin{array}{ll} 
& \underset{\substack{\left\{z_{i}, y_{i}\right\}, K, n, H \\
i=1, \ldots, N}}{\operatorname{aax}} u\left(z_{1}, G(n, K)\right), \\
\text { s.t. } \quad \text { (i) } \quad u\left(z_{i}, G(n, K)\right) \geq v_{i}, \quad i=2,3, \ldots, N \\
\\
\text { (ii) (2); } \quad z_{i} \geq 0, K \geq 0, n \in X, y_{i} \in Y_{i} \subset H, \quad i=1,2, \ldots, N .
\end{array}
$$

This states the IP optimality problem as one of trying to maximize the well-being of household 1 subject to required utility levels for others in the economy (constraint (i)) and feasibility (equation (2)). 
In the existing literature, an "optimal" configuration of public facilities is based on the concept of "command optimality" in which the community government determines the public facility configuration subject to (i) the competitive determination of the land rents (that often exit the system) and (ii) locational equilibrium (that equalizes households' utility). It is clear that such a concept is not well-defined in the Pareto sense - indeed, it is a mix of equilibrium and optimality concepts. ${ }^{13}$ To be compatible with the literature, however, we modify the concept of IP optimality by considering equal treatment (ET) among all households. Specifically, an equal-treatment identical-provision optimum (in short, an ETIP optimum) is an IP optimum such that all households reach the same utility, i.e., we have $u\left(z_{i}\left(x_{i}\right), G(n, K)\right)=v_{\mathrm{o}} \geq 0$ for all i $=1, \ldots, N$ in problem (4).

Two comments are now in order. First, an ETIP optimum is Pareto optimal provided that the provision of public good at all facilities is the same in an equal utility Pareto optimum and the number of consumers served at each facility is the same at the Pareto optimum. That is, an ETIP optimum may fail to be first-best if it is welfare enhancing to alter the ETIP optimum to serve different numbers of consumers or provide different levels of local public goods at different sites. As discussed in Section 5 below, there may be an allocation with non-identical public good provision that Pareto dominates the allocations restricted to identical provision. Second, our definition of ETIP optimality basically determines the optimal distribution to consumers for given aggregate endowments of the composite good and land ( $E$ and $L$, respectively), as well as optimal public facility configurations in terms of the number and locations of sites. Thus, it does not involve an equilibrium concept, contrasting with the command optimality concept in previous studies.

\section{Existence of an Equal-Treatment Identical Provision Optimum}

In this section we establish theorems concerning the existence of an ETIP optimum and its general

\footnotetext{
${ }^{13}$ This problem has been pointed out by Berliant and ten Raa (1994, p. 638): "Most of the models [of public facility location] appear to use a mix of positive and normative concepts, equilibria and optima. At best, they can be interpreted as partial welfare analysis."
} 
properties. An ETIP optimum can be obtained by solving the problem specified in (4).

To begin, we determine the optimal allocation between the public and the private composite good (see the Appendix for details of the calculations): $\sum_{i=1}^{N}\left(\frac{\partial u_{i} / \partial G}{\partial u_{i} / \partial z_{i}}\right) \frac{\partial G}{\partial K}=1$, which is in essence the Lindahl-Samuelson condition. With regard to ETIP optimality, we define $\Lambda(K, n, z) \equiv \frac{1}{N} \frac{\partial u / \partial z}{(\partial u / \partial G)(\partial G / \partial K)}$ and show that the ETIP optimal provision of the local public good satisfies the following necessary condition (see the Appendix):

$$
\Lambda(K, n, z)=1
$$

Denote $M R S_{G z} \equiv(\partial u / \partial G) /(\partial u / \partial z)$ as the marginal rate of substitution between the public and the private composite goods. It may be more intuitive to use (3) to rewrite (5) as: $\Lambda(K, n, z)=\frac{N^{\alpha} n^{1-\alpha}}{N\left(M R S_{G z}\right)}=1$. That is, the sum of the marginal willingness-to-pay (i.e., $N\left(M R S_{G z}\right)$, under ETIP) is equal to the marginal cost of public good provision (i.e., the net congestion factor $N^{\alpha} n^{1-\alpha}$ ). Notably, the optimal composite good allocation, the optimal number of public facility sites and the optimal level of the public good are all jointly determined, making it difficult to characterize the ETIP optimum. We thus take the method adopted by Bergstrom and Cornes (1983), seeking a condition such that the optimal level of public good provision is not only uniquely determined but also independent of the public facilities configuration and the distribution of composite good. By examining (5), we impose:

Condition K: $\Lambda(K, \mathrm{n}, \mathrm{z})$ is strictly increasing in $K$ with $\partial \Lambda / \partial \mathrm{n}=\partial \Lambda / \partial \mathrm{z}=0$.

Condition $\mathrm{K}$ is sufficient for (5) alone to pin down the optimal level of $K$, independent of $\mathrm{n}$ and $\mathrm{z}$.

Lemma 1: Under Assumptions (A2) and (A3) and Condition K, an ETIP optimum is associated with a uniquely determined level of public good that is independent of the public facility configuration.

Proof: Since $\partial \Lambda / \partial \mathrm{n}=\partial \Lambda / \partial z=0$, we can now write $\Lambda=\Lambda_{0}(K)$ with $\mathrm{d} \Lambda_{0} / \mathrm{d} K>0$. Thus, (5) can be inverted to determine the optimal provision $K=K_{0}$ where $K_{0} \equiv \Lambda_{0}^{-1}(1)$ is independent of $\mathrm{n}$ and $z$. The second-order 
condition is ensured by differentiating (5) and applying the condition, $\partial \Lambda / \partial K>0$.

Q.E.D.

To guarantee that the feasible set is non-empty, we consider:

Condition E: $K_{0}<E$.

For illustrative purposes, an example is now in order:

Example: Consider $u(z, G)=\gamma z+\ln (G)$ where $\gamma>0$. Then we have $\Lambda=\gamma K / N$ and hence (5) implies $K=$ $K_{0} \equiv N / \gamma$, independent of any endogenous variables. It is clear that Condition $\mathrm{E}$ is met if $\gamma(E / N)>1$, which requires households' valuation of the average composite good endowment be greater than one.

Due to the endogenous number of public facilities, Bergstrom and Cornes' (1983) condition of a separable utility function that is quasi-linear in the composite good is insufficient in our model to guarantee an optimal level of aggregate public good provision independent of other endogenous variables. The imposition of Condition K simplifies the analysis greatly.

Under Assumptions (A1)-(A3) and Conditions K, we can obtain another important necessary condition governing households' travel patterns for the optimal n (see the Appendix for details):

$$
\sum_{i=1}^{N} T\left(d\left(x_{i}, y_{i}\right)\right) \leq \sum_{i=1}^{N} T\left(d\left(x_{i}, y_{i}^{\prime}\right)\right), \quad \forall y_{i} \in Y_{i} \subset H, y_{i}^{\prime} \in Y_{i}^{\prime} \subset H^{\prime} \neq H
$$

Equation (6) says that given the optimal number of facilities, an optimal set of sites must be such that the aggregate travel cost is minimized. This condition is crucial for determining households' travel patterns as well as the optimal configuration of public facilities.

In an ETIP optimum, public good service is uniform across households (as specified in (3)) and thus the composite good consumption must be identical. Let the total transportation cost be denoted by $C(n, H) \equiv \sum_{i} T\left(d\left(x_{i}, y_{i}\right)\right)$, which is clearly non-increasing in n. The ETIP optimal composite good 
allocation to each household is then:

$$
z=(E-K-C) / N
$$

Since $C$ is a function of $(\mathrm{n}, \mathrm{H}), G$ is a function of $(\mathrm{n}, K)$ and, from Lemma $1, K=K_{0}$ (an exogenous constant), households' utility must be a function of $(\mathrm{n}, \mathrm{H})$ alone. We denote this value by $\mathrm{v}(\mathrm{n}, \mathrm{H})$.

Theorem: (Existence of ETIP Optimum) Under Assumptions (A1)-(A3) and Conditions $K$ and E, there exists an equal-treatment identical-provision optimum.

Proof. Denote the set of feasible allocations as $\mathrm{A}=\left\{\left(\left\{z_{\mathrm{i}}, Y_{\mathrm{i}}\right\}_{\mathrm{i}=1, \ldots, \mathrm{N}},\left\{K_{\mathrm{j}}, M_{\mathrm{j}}\right\}_{\mathrm{j}=1, \ldots, \mathrm{n}}, \mathrm{n}, \mathrm{H}\right) \mid\left\{z_{\mathrm{i}}, Y_{i}\right\}_{\mathrm{i}=1, \ldots, \mathrm{N}}\right.$ and $\left\{K_{\mathrm{j}}\right\}_{\mathrm{j}=1, \ldots, \mathrm{n}}$ satisfies (2), $Y_{i} \subset \mathrm{H} \forall \mathrm{i}=1, \ldots, N, \quad \sum_{j=1}^{n} M_{j}=N, \quad \mathrm{n}=\mu(\mathrm{H}) \in X$ and $\left.\mathrm{H}=\left\{\eta_{1}, \ldots, \eta_{\mathrm{n}}\right\} \subset \mathrm{L}\right\}$. Under (A1) and noting that $\mu(X)=N$ is finite, it is obvious that A is compact (closed and bounded). We then show that the set of IP feasible allocations is closed. Denote the set of IP feasible allocations as $\mathrm{P}=\left\{\left(\left\{z_{\mathrm{i}}\right.\right.\right.$, $\left.\left.Y_{\mathrm{i}}\right\}_{\mathrm{i}=1, \ldots, \mathrm{N}},\left\{K_{\mathrm{j}}, M_{\mathrm{j}}\right\}_{\mathrm{j}=1, \ldots, \mathrm{n}}, \mathrm{n}, \mathrm{H}\right) \in \mathrm{A} \mid$ each site $\eta_{\mathrm{j}} \in \mathrm{H}$ has a local public good of size $K_{\mathrm{j}}=K / \mathrm{n}$ and $\left\{Y_{\mathrm{i}}\right\}_{\mathrm{i}=1, \ldots, N}$ is consistent with $M_{\mathrm{j}}=\mathrm{N} / \mathrm{n}$ for all $\left.\mathrm{j}=1, \ldots, \mathrm{n}\right\}$. Since the IP restrictions only involve quantities $\mathrm{n}, y_{i} \in Y_{\mathrm{i}}$ $(\mathrm{i}=1, \ldots, N)$ and $\eta_{\mathrm{j}} \in \mathrm{H}(\mathrm{j}=1, \ldots, \mathrm{n})$ which all take integer values from 0 to $N$, the closedness property is trivial. Thus, $\mathrm{P}$ is compact as it is a closed subset of a compact set A. Further denote the set of ETIP feasible allocations as $\mathrm{F}=\left\{\left(\left\{z_{\mathrm{i}}, Y_{\mathrm{i}}\right\}_{\mathrm{i}=1, \ldots, \mathrm{N}},\left\{K_{\mathrm{j}}, M_{\mathrm{j}}\right\}_{\mathrm{j}=1, \ldots, \mathrm{n}}, \mathrm{n}, \mathrm{H}\right) \in \mathrm{P} \mid u\left(z_{i}, G(n, K)\right)=v_{\mathrm{o}} \geq 0\right.$ for all $\left.\mathrm{i}=1, \ldots, N\right\}$. We claim that $\mathrm{F}$ is closed. Under IP and Condition $\mathrm{K}$, it is sufficient to focus on continuous quantities $Z=\left(z_{1}, \ldots, z_{N}, K\right)$ in the ET restriction where $K=K_{0}$ by Lemma 1 . Consider a sequence of ETIP allocations $a^{\ell}=\left(\left\{z_{\mathrm{i}}^{\ell}, Y_{\mathrm{i}}\right\}_{\mathrm{i}=1, \ldots, \mathrm{N}},\left\{K_{0}, M_{\mathrm{j}}\right\}_{\mathrm{j}=1, \ldots, \mathrm{n}}, \mathrm{n}, \mathrm{H}\right) \in \mathrm{F}$ for all $\ell=0,1,2, \ldots$ and it has a limit point $a=\left(\left\{z_{\mathrm{i}}, Y_{\mathrm{i}}\right\}_{\mathrm{i}=1, \ldots, \mathrm{N}}\right.$, $\left.\left\{K_{0}, M_{\mathrm{j}}\right\}_{\mathrm{j}=1, \ldots, \mathrm{n}}, \mathrm{n}, \mathrm{H}\right) \in \mathrm{P}$. By construction, $u\left(z_{i}^{\ell}, G\left(n, K_{0}\right)\right)=v_{\mathrm{o}} \geq 0$ for all $\mathrm{i}=1, \ldots, N$. Since $u$ is continuous by (A3), we have $\left.u\left(z_{i}\right), G\left(n, K_{0}\right)\right)=v_{\mathrm{o}}$ and hence $a \in \mathrm{F}$, which proves the assertion. As $\mathrm{F}$ is a closed subset of a compact set $\mathrm{P}, \mathrm{F}$ is compact.

Next, we show that $\mathrm{F}$ is nonempty. It is obvious that by constructing an IP public facility configuration $(\mathrm{n}, \mathrm{H})$ with $\mathrm{n}=N, y_{i}=\eta_{\mathrm{i}} \in x_{i}, K_{\mathrm{i}}=K_{0} / N$ and $\mathrm{M}_{\mathrm{i}}=1(\mathrm{i}=1, \ldots, N)$, the corresponding total 
transportation cost is $C(\mathrm{n}, \mathrm{H})=0$ and $G\left(n, K_{0}\right)=K_{0} / N$. Under Condition E, we have $E-K_{0}-C(\mathrm{n}, \mathrm{H})>0$ and by choosing $z_{i}=\left(E-K_{0}\right) / N$, such allocations are ETIP feasible, implying $\mathrm{F} \neq \phi$.

Because $\mathrm{n}, \mathrm{y}_{\mathrm{i}} \in Y_{\mathrm{i}}(\mathrm{i}=1, \ldots, N)$ and $\eta_{\mathrm{j}} \in \mathrm{H}(\mathrm{j}=1, \ldots, \mathrm{n})$ are discrete and finite and $u$ is continuous for each fixed $\left(\left\{Y_{i}\right\}_{\mathrm{i}=1, \ldots, N}, \mathrm{n}, \mathrm{H}\right)$, the ETIP optimality problem has a continuous objective over a compact and nonempty domain F. By straightforward application of the Maximum Value Theorem implied by the Bolzano-Weierstrauss Theorem (Bartle (1976), pp. 154-155), an ETIP optimum exists. Q.E.D.

It is apparent that in establishing the theorem, all we need are the continuity of the households' utility function and the transport cost function, together with Condition $\mathrm{E}$ and the assumption that given the monotone increasing properties of $u$ in $G$ and $T$ in $d\left(x_{\mathrm{i}}, y_{i}\right), G$ is non-increasing in n (this latter condition ensures that $\mathrm{n}$ is finite, bounded by $N$ ). The assumptions regarding differentiability, specific functional forms, or interior solutions imposed in (A1)-(A3) are not necessary, but are made for a complete characterization of the ETIP optimum (in section 4 below).

Remark 1: Recall that $H=\left\{\eta_{1}, \ldots, \eta_{n}\right\}$ and hence $H$ and $n$ are inter-related. For each $n$, define $\mathrm{H}^{*}(n)=\operatorname{argmin}_{H} C(n, H)$. While the total transportation cost $C\left(n, H^{*}(n)\right)$ is non-increasing in n, the net congestion factor $N^{\alpha} n^{1-\alpha}$ is strictly increasing in $\mathrm{n}$ (for $\alpha<1$ ). Thus, under assumption (A3), equations (7) and (3) together with Lemma $1\left(K=K_{0}\right)$ imply that $z$ is non-decreasing in $\mathrm{n}$ and $G$ is strictly decreasing in $\mathrm{n}$. An immediate consequence of these conflicting effects on $u(z, G)$ is that an ETIP optimum may be associated with an intermediate value of $\mathrm{n} \in\{2, \ldots, N$-1 $\}$, i.e., an ETIP optimal public facility configuration need not be completely concentrated $(n=1)$ or completely dispersed $(n=N)$.

Remark 2: There are in general a continuum of ETIP optimal public facility configurations, even when $\mathrm{n}$ is fixed. For example, consider the case of $\mathrm{n}=1$ and $N$ as an odd number with a central region [ [N/2], $[N / 2]+1]$, where [D] is the Gauss operator of $\mathrm{D} \in \mathbb{R}_{+}$, defined as the largest integer that is less than or equal 
to D. We will show in Section 4 below that under assumptions (A1)-(A3) and Conditions K and E, any $\eta \in$ [ [N/2], [N/2]+1 ] is ETIP optimal. When $\mathrm{n}=1$ and $N$ is an even number, there is a unique ETIP optimum $\eta$ $=N / 2$, which is in between the front door of the site of [N/2]-household and the back door of the site of ([N/2]+1)-household.

We would like to remind the reader that the main purpose of this paper is to perform a normative analysis of local public facility configuration, which contrasts sharply with the existing literature based on a positive analysis of command optimality. Nevertheless, for comparison, we focus primarily on ETIP allocations, which are consistent with previous studies.

\section{Characterization of Optimal Public Facility Configurations}

We characterize ETIP optimal public facility configurations explicitly given a linear transportation cost schedule specified as in (1). We focus on three issues: (i) whether an ETIP optimal configuration is uniquely determined, (ii) whether public facilities should be concentrated in one location or dispersed in more than one location, and (iii) whether a concentrated public facility should be geographically centralized.

\subsection{Optimal Location of Public Facility: The Case of $\mathbf{n}=\mathbf{1}$}

It is important to note that the uniqueness/multiplicity properties of ETIP optimality depend crucially on the number of households. First we fix the number of facilities exogenously at 1 .

Proposition 1: (ETIP optimal location of public facility with $\mathrm{n}=1$ ) Assume (A2), (A3), Conditions K and E, and the linear transportation cost schedule specified as in (1). Given $n=1$, the set of ETIP optimal public facility configurations satisfies the following properties:

(i) for even numbers $N$, it is unique and geographically centralized;

(ii) for odd numbers $N>1$, it is a continuum consisting of any location in the central residential interval. 
Proof: To prove part (i), consider an even number of households $N=2$. As shown in Figure 1, for given unit transportation cost $t>0$, we start with $\eta=2$ (for the case of $\eta=0$, the arguments are similar and hence omitted). In this case, the total transportation cost is $C=t$. Now, we move the public facility to $\eta=1$. While the local public good service $G$ is unchanged, the total composite good available for allocation to households increases from $E-K_{0}-t$ to $E$ - $K_{0}$, resulting in a Pareto improvement. Similar arguments apply to any $\eta \in(1,2)$. Therefore, the allocation $\left(z_{i}, K, n, H\right)=\left(E / N-K_{0} / 2, K_{0}, 1,\{1\}\right)$ is ETIP optimal, where $K_{0}$ solves (5). The corresponding total transportation cost schedule with respect to the public facility location is depicted in bottom panel of Figure 1 from which one can see that $\eta=1$ is associated with minimum total transportation costs, satisfying the requirement in (8). This analysis and the uniqueness result apply to the case with $\mathrm{n}=1$ and any even number $N$.

We next prove part (ii) by considering $N$ odd. We claim that any allocation with $H=\{\eta\}$ where $\eta$ $\in[[N / 2],[N / 2]+1]$ (i.e., the central region) is ETIP optimal. Suppose not. Consider the public facility site on the right of the central region with $\eta=[N / 2]+1+\epsilon$, where $\epsilon>0$. Then moving the site to $\eta=[N / 2]+1$ would result in an improved allocation as it reduces the total transportation cost by $2 \epsilon t$. Similar arguments apply for the case with the public facility site on the left of the central region and for any $\epsilon>0$. Next, we show that the relocation of the public facility site within the central region leaves the total transportation cost unchanged. Consider moving the site from $\eta=[N / 2]+1$ to $\eta=[N / 2]+1-\epsilon$, where $\epsilon \in(0,1)$. While the transportation cost of each household residing on the left of the central region is reduced by $\epsilon t$, that of each household on the right is raised by $\epsilon t .{ }^{14}$ Since the total transportation $\operatorname{cost} C$ is unchanged, these allocations are all ETIP optimal.

Q.E.D.

The results in Proposition 1 contrast with findings in the framework with a continuum of households of Fujita (1986) and Sakashita (1987) in two important dimensions. First, in our model, an ETIP optimal

\footnotetext{
${ }^{14}$ For illustrative purposes, we delineate the case of $N=3$ and $\epsilon=1 / 2$ in Figure 2(a).
} 
location of the public facility need not be at the geographic center. Second, we find that multiple ETIP optimal configurations are possible in the sense that the optimal location of the public facility need not be unique and need not be geographically centralized. These discrepancies are mainly due to having a finite number instead of a continuum of households. It may be noted that as $N$ goes to infinity (by expanding L to the entire extended real line), this multiplicity property is still robust. One may argue that if we fix the landscape of the entire residential region but take $N$ to infinity, the optimal location of public facility may reduce to a single point at the geographical center. However, such an exercise is mathematically inaccurate because the individual household lot size would approach zero (see Berliant and ten Raa 1991).

\subsection{Optimal Number and Locations of Public Facilities: the Case of $N=3$}

We next turn to endogenizing the number of public facilities. When $N \geq 3$, it is possible that the ETIP optimal number of public facilities is no longer one. For illustrative purposes, it suffices to consider the case of $N=3$. Given $\alpha<1$ (i.e., $G$ is not a pure private good), we claim:

Lemma 2: Assume (A2), (A3), Conditions $K$ and $E$, and the linear transportation cost schedule specified as in (1). Given $N=3$, the ETIP optimal number of public facilities $n$ can only be 1 or 2.

Proof: This lemma can be easily proved by contradiction. Suppose $\mathrm{n}=3$ is ETIP optimal with one public facility of size $K_{0} / 3$ in each residential interval (so no one commutes). Thus, $G\left(3, K_{0}\right)=K_{0} / 3$. Consider an alternative ETIP allocation with $\mathrm{n}=2$ and $\mathrm{H}=\{1,2\}$ in which $C(2,\{1,2\})=0$. Now the public good service becomes $G\left(2, K_{0}\right)=(3 / 2)^{1-\alpha}\left(K_{0} / 3\right)$, strictly greater than $K_{0} / 3$ for any $\alpha<1$. Thus, an allocation with $\mathrm{n}=3$ cannot be an ETIP optimum.

Lemma 2 rules out the possibility of $n=3$. Hence, there are three candidate ETIP optimal public facility configurations remaining to be examined with $N=3$ : (i) centrally concentrated (type-C), (ii) noncentrally concentrated (type-N) and (iii) dispersed (type-D). Notably, Proposition 1 implies that an ETIP 
optimum with $\mathrm{n}=1$ (concentrated) must have the public facility of size $K_{0}$ in the central residential interval. In this case, there are two possibilities: either $H=\{3 / 2\}$ (i.e., type-C) or $H=\{\eta\}$ with $\eta \in[1,2] \backslash\{3 / 2\}$ (i.e., type-N). With regard to the dispersed case $(n=2)$, we claim:

Lemma 3: Assume (A2), (A3), Conditions $K$ and $E$, and the linear transportation cost schedule specified as in (1). Given $N=3$, if the type-D public facility configuration is ETIP optimal, there are two locationally symmetric and identically provided public facilities of size $K_{0} / 2$ located at $\eta=1$ and 2 .

Proof: To prove this lemma, we utilize Figure 2(b). In particular, we need to show that public facilities must be located at 1 and 2. Suppose not. Consider, say, an alternative ETIP feasible allocation with $\mathrm{H}=$ $\{1 / 2,5 / 2\}$. Then using the arguments in the proof of Proposition 1, household 2 may have an improving reallocation with $H=\{1,2\}$, leaving households 1 and 3 unaffected. Since this reallocation is ETIP feasible but better to household 2 (where ET can be achieved with $z=\left(E-K_{0}\right) / 3$ ), it contradicts the definition of ETIP optimality. By similar arguments, one can show that any other ETIP feasible allocations with $\mathrm{n}=2$ and $H \neq\{1,2\}$ is Pareto dominated by that with $H=\{1,2\}$ as the latter is the only ETIP feasible allocation associated with zero total transportation cost.

Q.E.D.

We are now left to examine each candidate configuration and to derive the corresponding conditions to support each case. In order to compute household's indirect utility, we further assume that the utility function of the households takes the following form: ${ }^{15}$

$$
u(z, G)=\gamma z+\ln (G)
$$

where $\gamma>0$. From Lemma 1, we can solve the optimal provision of the local public good as $K_{0}=N / \gamma$. Utilizing Lemmas 2 and 3, we can substitute the composite good allocation (7), and the public good service

${ }^{15}$ Those interested in the conventional Cobb-Douglas utility functional form are referred to the Appendix where one must assume the level of the public good provision $K$ is exogenously fixed. As one can easily see below, the main conclusions are robust to functional form. 
(3) under each type into the utility function (8) to obtain the household value for each type of public facility configuration as follows:

$$
\begin{gathered}
v^{C}=v^{N}=\gamma\left(E-K_{0}\right)+\ln \left(K_{0} / 3\right)+(1-\alpha) \ln 3-\gamma t \\
v^{D}=\gamma\left(E-K_{0}\right)+\ln \left(K_{0} / 3\right)+(1-\alpha) \ln 3-(1-\alpha) \ln 2
\end{gathered}
$$

where the superscripts denote the types of public facility configurations and the corresponding aggregate transportation costs are: $\quad C^{C}=C^{N}=t \quad$ and $\quad C^{D}=0$.

To compare different types of public facility configurations, we need to check if they are Pareto rankable. This is easily done by comparing households' value for each case, as given by (9a) and (9b).

Proposition 2: (ETIP optimal location of public facility with $N=3$ ) Assume (A2), Conditions $K$ and $E$, the linear transportation cost schedule specified as in (1), and the utility function specified as in (8). When $N$ = 3, an ETIP optimal public facility configuration satisfies the following properties:

(i) if $\mathrm{t}>(\ln 2)(1-\alpha) / \gamma$, then an ETIP optimal public facility configuration is always dispersed;

(ii) if $\mathrm{t}<(\ln 2)(1-\alpha) / \gamma$, then an ETIP optimal public facility configurations is always concentrated, either centralized or non-centralized.

Proof: The proof follows immediately from comparing (9a) and (9b):

$$
v^{C}=v^{N} \underset{<}{<} v^{D} \Leftrightarrow t \frac{<}{>}(\ln 2)(1-\alpha) / \gamma
$$

provided that $\gamma E>3$, which ensures Condition $E$ is met.

Q.E.D.

From (10), the determination of the public facility configuration depends crucially on the transportation cost $(t)$, the degree of non-exclusiveness $(1-\alpha)$, and the household valuation of the public good 
$(1 / \gamma)$. Therefore, within our congestable local public good framework with $\alpha<1$, we can conclude from Proposition 2,

Proposition 3: Assume (A2), Conditions $K$ and $E$, the linear transportation cost schedule specified as in (1), and the utility function specified as in (8). Given $N=3$, an ETIP optimal public facility configuration is dispersed if the degree of congestability is large, the household valuation of the public good is low, and the unit transportation cost is sufficiently high; otherwise, an ETIP optimal public facility configuration is concentrated.

Therefore, building a large central park (or library, or school) is not ETIP optimal in an economy with a high degree of public good congestability or a high transportation cost. A Pareto improvement results if the public good service is provided at dispersed facility sites with smaller size. This justifies the existence of local community parks, libraries and schools in reality.

Our results can be compared with those in previous studies. Sakashita (1987) considers the case of two pure public good facilities (although this number is exogenously given therein). He finds that in the absence of mutual accessibility between the two public facilities, the two public facility sites are at the first and third quarter of the linear city. In our model with $N=3$, when the (endogenously determined) ETIP optimal configurations are dispersed, the optimal public facility sites are at one-third and two-thirds in the linear city.

In another related paper, Hochman and Pines (1997) compare economic welfare with one or two public facilities given two distinct pure public goods in a model with a continuum of households. They conclude that two facilities (one for each pure public good) are optimal when the unit transportation cost is extremely large or extremely small. Our results suggest, however, that a dispersed configuration is optimal only when the unit transportation cost is sufficiently large. Moreover, it is worth noting that the optimality concept in their paper is completely different from our concept of ETIP optimum. Specifically, they consider 
an open model with an endogenous population of households, in which the welfare of those not within the model is ignored and hence Pareto optimality is not well-defined.

\subsection{Optimal Number and Locations of Public Facilities: the General Case of $N>3$ and $\mathbf{n} \geq \mathbf{1}$}

In Section 4.1, we considered the case $\mathrm{n}=1$ for any given $N$, whereas Section 4.2 examines the case of $N=3$ for an endogenous determination of ETIP optimal $\mathrm{n}$ that need not be equal to one. ${ }^{16} \mathrm{We}$ now characterize the general case of $N>3$ allowing for multiple public facility sites.

We begin by proving the following Lemma:

Lemma 4: Assume (A2), Conditions $K$ and $E$, the linear transportation cost schedule specified as in (1), and the utility function specified as in (8). In an ETIP optimum, the following properties always hold:

(i) for any even number $N$, there should be no more than N/2 public facilities;

(ii) for any odd number $N$ that is a multiple of 3, there can be no more than 2N/3 public facilities;

(iii) for an odd number $N$ that is not a multiple of 3 , there may be as many as $N$ public facilities.

Proof: The proof of parts (i) and (ii) follows arguments similar to the proof of Lemma 2. When $N$ is an even number, it is always Pareto dominant to have at least two adjacent households sharing one public facility at their back/front door location in which the public good service is higher than that with one public facility in each of the two residential regions. Both allocations incur zero total transportation cost. When $N$ is an odd number that is a multiple of 3 , a configuration with three connected households sharing two public facilities as illustrated in Figure 2(b) always Pareto dominates the completely dispersed case with one public facility in each residential region.

For part (iii), it is sufficient to consider the case of $N=5$. Since equal numbers of people must be served at all sites, there are three possible IP numbers of public facilities: $n=1,2$ and 5 . It is easily seen

${ }^{16}$ The case of $N=2$ is trivial: the ETIP optimal configuration must be $\mathrm{n}=1$ and $\eta=1$ (concentrated and centralized). It is therefore omitted from our discussion. 
that if $K_{0}<E<K_{0}+t$ (a sufficient but not necessary condition), the ETIP optimal configuration of public facilities must be associated with $n=5$ (since any allocation with $n=1$ or 2 is not IP feasible). These arguments together with Lemma 2 imply that for an odd number that is not a multiple of 3, it is possible to have an ETIP optimal configuration with $\mathrm{n}=N$ if the transportation cost is sufficiently large relative to the aggregate endowment.

Q.E.D.

Notice that part (iii) of Lemma 4 is due in particular to the considerations of identical local public good provision. For example, it is impossible to have an IP feasible allocation with $n=3$ or 4 in the case of $N=5$; otherwise, the number of consumers patronizing each site cannot be the same. ${ }^{17}$ This therefore makes the completely dispersed $(\mathrm{n}=N=5)$ public facility configuration possible. In general, for any number $N>$ 3, there always exists an IP public facility configuration of $\mathrm{n}=1,2$ and $N$. However, a completely dispersed public facility configuration is ETIP optimal only if $N$ is not a multiple of 2 or 3 .

Before establishing the general proposition for $N>3$ in Lemma 5 and Proposition 4 below, we would like to make two remarks. First, with regard to concentrated $(n=1)$ versus dispersed $(n>1)$ public facility configurations, we claim that Proposition 3 can be applied to the general case of $N>3$. In particular, an ETIP optimal public facility configuration is always dispersed when there is a high degree of public good congestability, it is costly to commute and household valuation of the public good service is low. Otherwise, there are multiple ETIP optimal public facility configurations where the optimal configurations could be concentrated or dispersed. This characterization is robust with respect to the number of households $N>3$.

Second, it may be interesting to examine the case of two optimal public facility sites $(n=2)$ to compare with Sakashita's (1987) result (assuming an appropriate set of parameters making n = 2 optimal: for example, $t>(\ln 2)(1-\alpha) / \gamma \quad$ under the utility function specification in (8) for $N=3)$. Recall that Sakashita's optimal public facility location at the first and third quarter of the landscape does not hold in our

17 We relegate the discussion of non-IP feasible allocations to Section 5 below. 
model with $N=3$ (the ETIP optimal sites are at one third and two thirds). Yet Sakashita's result may occur in our model with $N=4$ or any numbers that are multiples of 4 . To obtain a general property, let us study the cases of $N=5,6,7,9$ and 10. When $N=5$, the ETIP optimal configuration is $\mathrm{H}=\{1,4\}$. For $N=6$, we have $H=\left\{\eta_{1}, \eta_{2}\right\}$ with $\eta_{1} \in[1,2]$ and $\eta_{2} \in[4,5]$; for $N=7, H=\{2,5\}$; for $N=9, H=\{2,7\}$; and for $N=10, \mathrm{H}=\left\{\eta_{1}, \eta_{2}\right\}$ with $\eta_{1} \in[2,3]$ and $\eta_{2} \in[7,8]$. For $N=5,7$ and 9 , Sakashita's first and thirdquarter configuration is not ETIP optimal. For the cases of $N=6$ and $N=10$, Sakashita's first and thirdquarter configuration is only one of the ETIP optimal outcomes. Thus, his finding is not robust in general.

More generally, we can characterize ETIP locations of public facilities for $N>3$ given the linear transport cost schedule as in (1) and the utility function specification as in (8). For illustrative purposes, we focus on a set of parameters such that an ETIP optimum is featured with $n=2$. We claim:

Lemma 5: Assume (A2), Conditions $K$ and $E$, the linear transportation cost schedule specified as in (1), and the utility function specified as in (8). Given $N>3$, the set of parameters resulting in an ETIP optimal number of public facilities equal to two is nonempty.

Proof: Recalling that ${ }^{*}(n)=\operatorname{argmin}_{H} C(n, H)$, substituting (3), (7) and $K=K_{0}$ into (8) and equating the utility values associated with $\mathrm{n}=1$ and $\mathrm{n}=2$, for $N>3$, yield: $C\left(1, H^{*}(1)\right)-C\left(2, H^{*}(2)\right)=(\ln 2)(1-\alpha) / \gamma$. It is easily shown that for $\mathrm{N}=4, \mathrm{C}\left(1, H^{*}(1)\right)-C\left(2, H^{*}(2)\right)=2 t$. Define $I_{N} \equiv\left\{2+\sum_{J=5}^{N}\left(\left[\frac{J-3}{2}\right]-\left[\frac{J-5}{4}\right]\right)\right\}$. By induction, we can derive $C\left(1, H^{*}(1)\right)-C\left(2, H^{*}(2)\right)=t I_{N}$ for all $N \geq 5$. Let $\hat{t}=(\ln 2)(1-\alpha) /\left(\gamma I_{N}\right)$. One can then see that, for any $t$ with $t-\hat{t}$ approaching to zero from above, $\mathrm{n}=2$ is ETIP optimal. $\quad$ Q.E.D. Utilizing Lemma 5, we can show:

Proposition 4: Assume (A2), Conditions $K$ and $E$, the linear transportation cost schedule specified as in (1), and the utility function specified as in (8). Given $N>3$ and a set of parameters such that $\operatorname{argmin}_{n} C\left(n, H^{*}(n)\right)=2$, ETIP optimal locations of public facilities possess the following properties: (i) when $N$ is a multiple of $4, H=\{N / 4,3 N / 4\}$; 
(ii) when $N$ is even but not a multiple of $4, H=\left\{\eta_{1}, \eta_{2}\right\}$ with $\eta_{1} \in[[N / 4],[N / 4]+1]$ and $\eta_{2} \in[N-$ $[N / 4]-1, N-[N / 4]]$

(iii) when $N$ is an odd number, $H=\{[(N+1) / 4], N-[(N+1) / 4]\}$.

Proof: By Lemma 5, the set of parameters yielding an ETIP optimal number of public facilities $\mathrm{n}^{*}=2$ is nonempty. Case (i) is trivial. For case (ii), divide the entire region evenly into two where each half has a public facility site. By symmetry, we focus on the left half, $\mathrm{L}_{1} \equiv[0, N / 2]$. Since $N$ is not a multiple of $4, \mathrm{~L}_{1}$ must contain an odd number of households. Since there is only one public facility in $\mathrm{L}_{1}$, we can apply Proposition 1 (ii) to conclude that the ETIP optimal site must be in the central residential interval in $\mathrm{L}_{1}$, which is [ [N/4], [N/4] +1 ]. By symmetry, the site in the right half is obtained.

Next, consider case (iii). Since it is always suboptimal to have both sites in the central region, we begin by removing the household residing in the central region (denoted $i_{m}$ ) and denoting $\mathrm{L}_{2} \equiv[0,[N / 2]]$ which must contain one of the two sites. There are now two subcases to investigate. First, if $\mathrm{L}_{2}$ contains an even number of households (i.e., [N/2] is even), by Proposition 1 (i), the ETIP optimal public facility location must be at the geographic center of $\mathrm{L}_{2}$. Then we add back the middle household $i_{m}$. By randomization, only half of that household involves commuting to the public facility site in $\mathrm{L}_{2}$, we claim that adding back $i_{m}$ would not change the optimal public facility location. Suppose not, say, the facility site shifts toward $i_{m}$ (it is trivial that a shift away from $i_{m}$ can never be optimal). In this case, the reduction of the transportation cost incurred by $i_{m}$ is half of the increased transportation cost by household 1, thus leading to a higher total transportation cost and contradicting the optimality condition. Therefore, the optimal location is at $\eta=[N / 2] / 2$. Second, if $\mathrm{L}_{2}$ contains an odd number of households (i.e., [N/2] is odd), from case (ii) above, the optimal location is the central interval of $\mathrm{L}_{2},[[N / 4],[N / 4]+1]$. Of course, when we add back $i_{m}$, only the upper bound of this interval is optimal, i.e., $\eta=[N / 4]+1$. Notice that by the same arguments as above, the optimal allocation would not shift over to the adjacent interval toward $i_{m}$. Combining the two subcases, we obtain the ETIP optimal location in $\mathrm{L}_{2}$ as $\eta=[(N+1) / 4]$ and that the analogous result holds for 
the right half as well.

Q.E.D.

It may be informative to study the limit properties of ETIP optimal allocation as $N$ approaches infinity. For case (iii), one learns that $[(N+1) / 4] / N \rightarrow 1 / 4$ and $(N-[(N+1) / 4]) / N \rightarrow 3 / 4$. However, the limit case of (ii) would not reduce to the first-quarter and third-quarter rule found in Sakashita (1987).

\section{On the Non-Identical Provision of the Local Public Good}

Up to this point, we have restricted our attention to the case of identical local public good provision. In this Section, we study the consequences of relaxing the IP assumption. In particular, we illustrate that a feasible allocation with non-identical provision of a local public good Pareto dominates any IP feasible allocations. That is, we claim:

Proposition 5: Assume (A2), Conditions $K$ and $E$, the linear transportation cost schedule specified as in (1), and the utility function specified as in (8). An ETIP optimum may be Pareto inferior to a non-identical provision feasible allocation.

Proof: It is sufficient to verify this by examining a special case of $N=5$ where there are three possible IP feasible allocations with $n=1,2$ and 5. Consider a non-IP feasible allocation with $n=3, H=\{1,5 / 2,4\}$ and the provision of the local public good at each site is $\left\{2 K_{0} / 5, K_{0} / 5,2 K_{0} / 5\right\}$, respectively, where $K_{0}=5 / \gamma$. It is obvious that no household incurs transportation cost and that households $1,2,4$, and 5 receive a higher level of public good service than household 3 (since $\alpha<1$ ). Denote household 3's indirect utility in this nonIP case of $\mathrm{n}=3$ as $v_{3 \mathrm{~L}}$ while that of household 1, 2, 4 and 5 as $v_{3 \mathrm{H}}\left(\right.$ where $v_{3 \mathrm{~L}}<v_{3 \mathrm{H}}$ ). Further denote the ET level of indirect utility in the IP cases of $\mathrm{n}=1,2$ and 5 as $v_{1}, v_{2}$ and $v_{5}$ respectively. Straightforward manipulations yield:

$$
v_{3 L}=v_{5}=\gamma\left(E-K_{0}\right)+\ln K_{0}-\ln 5
$$




$$
\begin{gathered}
v_{1}=\gamma\left(E-K_{0}\right)+\ln K_{0}-\alpha \ln 5-4 \gamma t \\
v_{2}=\gamma\left(E-K_{0}\right)+\ln K_{0}-\alpha \ln 5-(1-\alpha) \ln 2-\gamma t
\end{gathered}
$$

Consider,

$$
\left(\frac{1-\alpha}{\gamma}\right) \ln \left(\frac{5}{2}\right) \leq t
$$

From (11a) and (11c), (12) implies that $v_{5} \geq v_{2}$ given $t>0$ and $\alpha<1$. Under (12), it must be true that $t \geq[(1-\alpha) /(3 \gamma)] \ln 2$ and hence we can show from (11b) and (11c) that $v_{2} \geq v_{1}$. That is, condition (12) is sufficient to ensure the indirect utility ordering: $v_{3 \mathrm{~L}}=v_{5} \geq v_{2} \geq v_{1}$. Since households $1,2,4$, and 5 in the nonIP case described above receive strictly higher levels of utility than household 3 as long as $\alpha<1$, the non-IP allocation proposed above Pareto dominates any IP feasible allocations and hence any ETIP optima. Q.E.D.

\section{Concluding Remarks}

We have examined a model with a finite number of households and with a congestable local public good where the number and location of facility sites as well as the level of public good provision are endogenously determined. We prove the existence of an ETIP optimum. We show that there may be a continuum of ETIP optimal public facility configurations and an ETIP optimum public facility location need not be central geographically even if there is only a single public facility. An ETIP optimal public facility configuration must be dispersed with multiple facility sites if the degree of congestability and the unit transportation cost are high and the household valuation of the local public good is low. Otherwise, the ETIP optimal public facility configuration is concentrated.

Along these lines, there are at least three possible avenues of interest for future research. First, one may explore the theoretical front, examining the nonemptiness of an ETIP core (thus refining the set of ETIP optima) and the validity of the first and second welfare theorems based on Lindahl or competitive spatial 
equilibrium. Second, we may relax the assumption of identical households to allow for, say, two types of households, with heterogeneous endowments or tastes. We may study whether an optimal public facility is geographically biased toward the rich or a consumer who likes the public good. Finally, it is possible to consider more than one public good. When all public goods are necessities, it is interesting to examine whether a concentrated public facility configuration may still emerge where different public goods are provided in the same site. 


\section{Appendix}

\section{A. Derivation of ETIP Optima:}

To solve problem (4), we note that the travel pattern is the solution to a trivial distance minimization problem, independent of other choices and thus we set up the Lagrangian function as:

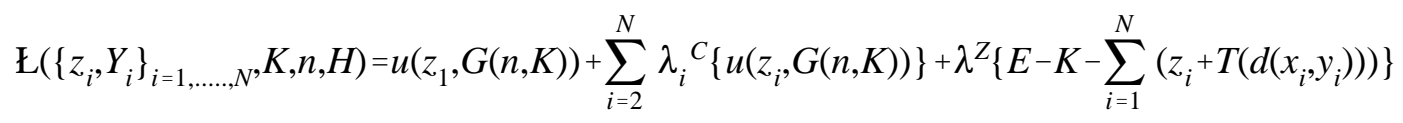

where $G(\mathrm{n}, K)=K /\left(N^{\alpha} \mathrm{n}^{1-\alpha}\right)$ and the multipliers $\lambda^{\mathrm{C}}$ and $\lambda^{\mathrm{Z}}$ are, in sequential order, associated with the two constraints given in (4).

The first-order conditions are:

$$
\begin{gathered}
\partial u_{1} / \partial z_{1}-\lambda^{Z}=0, \\
\lambda_{i}{ }^{C}\left(\partial u_{i} / \partial z_{i}\right)-\lambda^{Z}=0, \quad i=2, \ldots, N \\
\left(\partial u_{1} / \partial G\right)(\partial G / \partial K)+\sum_{i=2}^{N} \lambda_{i}^{C}\left(\partial u_{i} / \partial G\right)(\partial G / \partial K)=\lambda^{Z}, \\
\mathrm{七}\left(z_{i}, K, n, H\right) \geq \mathrm{E}\left(z_{i}, K, n^{\prime}, H^{\prime}\right), \quad \forall H^{\prime} \subset L, H^{\prime} \neq H, n^{\prime}=\mu\left(H^{\prime}\right) \in X,
\end{gathered}
$$

For any n, (A-2a) and (A-2b) together imply:

$$
\left(\partial u_{1} / \partial z_{1}\right) /\left(\partial u_{i} / \partial z_{i}\right)=\lambda_{i}^{C}
$$

Substituting (A-2a) and (A-3) into (A-2c) to eliminate $\lambda^{Z}$ and $\lambda_{i}{ }^{C}$, we obtain:

$$
\left(\partial u_{1} / \partial G\right)(\partial G / \partial K)+\sum_{i=2}^{N} \frac{\partial u_{1} / \partial z_{1}}{\partial u_{i} / \partial z_{i}}\left(\partial u_{i} / \partial G\right)(\partial G / \partial K)=\partial u_{1} / \partial z_{1}
$$


or, by rearranging terms,

$$
\sum_{i=1}^{N} \frac{1}{\partial u_{i} / \partial z_{i}}\left(\partial u_{i} / \partial G\right)(\partial G / \partial K)=1
$$

Under ETIP, all individuals have the same public good service $\mathrm{G}$ and receive $\mathrm{u}\left(z_{\mathrm{i}}, \mathrm{G}\right)=v_{0}$. Thus, $z_{\mathrm{i}}$ are the same for all i and (A-4b) becomes:

$$
N\left(\frac{\partial u / \partial G}{\partial u / \partial z}\right) \frac{\partial G}{\partial K}=1
$$

Define $\Lambda(K, n, z) \equiv \frac{1}{N} \frac{\partial u / \partial z}{(\partial u / \partial G)(\partial G / \partial K)}$. Then, equation (5) follows immediately from (A-5).

Next, from (A-2a), $\lambda^{Z}>0$, which together with (A-1) implies $\mathrm{\iota}\left(z_{i}, K, n, H\right) \quad$ is strictly decreasing in the aggregate transportation cost. Thus, the optimal choice of $y_{i}$ given by (A-2e) must satisfy:

$$
\sum_{i=1}^{N} T\left(d\left(x_{i}, y_{i}\right)\right) \leq \sum_{i=1}^{N} T\left(d\left(x_{i}, y_{i}^{\prime}\right)\right), \quad \forall y_{i} \in H, y_{i}^{\prime} \in H^{\prime} \neq H, \mu(H) \leq N, \mu\left(H^{\prime}\right) \leq N
$$

Under Condition E, (A-3) and (A-6) together with the second constraint of (4) jointly determine the optimal locations of public facilities and the indirect utility level $v(\mathrm{n}, \mathrm{H})$ for a given $\mathrm{n}$ with $K=K_{0}$. By comparing $v(\mathrm{n}, \mathrm{H})$ for each $\mathrm{n} \in \mathrm{X}$, the optimal number of public facilities $n$ is therefore pinned down.

\section{B. An Alternative Example: the Case of Cobb-Douglas Utility Function}

Assume instead that the utility function of the households takes the Cobb-Douglas form, as in the conventional literature where $K$ is fixed exogenously:

$$
u[z, G(n)]=z^{\gamma}[G(n)]^{1-\gamma},
$$

where $\gamma \in(0,1)$ with $1-\gamma$ capturing the household valuation of the public good. The exogeneity of $K$ is required since Condition $\mathrm{K}$ is no longer met under the Cobb-Douglas utility function specification. Consider $N=3$. Utilizing Lemmas 2 and 3, we can combine (3), (7) and (B-1) to yield: 


$$
\begin{gathered}
v^{C}=v^{N}=\frac{K^{1-\gamma}[(E-K)-t]^{\gamma}}{3^{\alpha(1-\gamma)+\gamma}} \\
v^{D}=\frac{K^{1-\gamma}(E-K)^{\gamma}}{2^{(1-\alpha)(1-\gamma)} 3^{\alpha(1-\gamma)+\gamma}} .
\end{gathered}
$$

where $C^{C}=C^{N}=t$ and $C^{D}=0$.

Define:

$$
B(\alpha, \gamma) \equiv 1-\left(\frac{1}{2}\right)^{(1-\alpha)\left(\frac{1-\gamma}{\gamma}\right)} \quad \in(0,1)
$$

which depends positively on the degree of non-exclusiveness $(1-\alpha)$ and household's valuation of the public $\operatorname{good}(1-\gamma)$. We then prove:

Proposition 2': (ETIP optimal location of public facility with $N=3$ ) Assume (A2), Conditions $K$ and $E$, the linear transportation cost schedule specified as in (1), and the utility function specified as in (B-1). When $N=3$, an ETIP optimal public facility configuration satisfies the following properties:

(i) if $B(\alpha, \gamma)(E-K)<t$, then an ETIP optimal public facility configuration is always dispersed;

(ii) if $B(\alpha, \gamma)(E-K) \geq t$, then an ETIP optimal public facility configuration is always concentrated, either centralized or non-centralized.

Proof: Comparing (B-2a) and (B-2b), one gets:

$$
v^{C}=v^{N} \frac{>}{<} v^{D} \Leftrightarrow B(\alpha, \gamma)(E-K) \frac{>}{<} t
$$

which completes the proof.

Q.E.D.

From (B-3), $B(\alpha, \gamma)$ depends positively on the degree of non-exclusiveness and the household valuation of the 
public good.

We next remind the reader that the characterization of the general case of $N>3$ with multiple public facility sites (i.e., the properties in Lemma 4 and Proposition 4) remain valid under the Cobb-Douglas utility function specification, so long as $K$ is exogenously fixed.

With regard to the non-identical provision of the local public good, we show that the properties in Proposition 5 still holds. This is done by following the arguments in the proof of Proposition 5, with (11a)(11c) replaced by:

$$
\begin{gathered}
v_{3 L}=v_{5}=K^{1-\gamma}(E-K)^{\gamma} / 5^{1-\gamma} \\
v_{1}=K^{1-\gamma}(E-K-4 t)^{\gamma} / 5^{\alpha(1-\gamma)} \\
v_{2}=K^{1-\gamma}(E-K-t)^{\gamma /\left[2^{(1-\alpha)(1-\gamma)} 5^{\alpha(1-\gamma)}\right]}
\end{gathered}
$$

Consider,

$$
\begin{aligned}
& {\left[1-\left(\frac{2}{5}\right)^{(1-\alpha)\left(\frac{1-\gamma}{\gamma}\right)}\right](E-K) \leq t} \\
& \frac{1-2^{(1-\alpha)\left(\frac{1-\gamma}{\gamma}\right)}}{1-4\left[2^{(1-\alpha)\left(\frac{1-\gamma}{\gamma}\right)}\right]}(E-K) \geq t
\end{aligned}
$$

It is trivial that the left-hand side of (B-6b) is strictly greater than the left-hand side of (B-6a) since $3>(2 / 5)^{(1-\alpha)(1-\gamma) / \gamma}$. Using (B-5a) and (B-5c), (B-6a) guarantees $v_{5} \geq v_{2}$; also, from (B-5b) and (B-5c), (B6b) ensures $v_{2} \geq v_{1}$. That is, under (B-6a) and (B-6b), $v_{3 \mathrm{~L}}=v_{5} \geq v_{2} \geq v_{1}$. Following similar arguments, the non-IP allocation proposed in the proof of Proposition 5 Pareto dominates any IP feasible allocations and hence any ETIP optima. 


\section{References}

Alonso, W., 1964, Location and Land Use, Cambridge, Harvard University Press.

Arnott, R.J. and J.E. Stiglitz, 1979, Aggregate Land Rents, Expenditure on Public Goods, and Optimal City Size, Quarterly Journal of Economics, 93, 471-500.

Asami, Y., M. Fujita and T. Smith, 1991, On the Foundations of Land Use Theory: Discrete Versus Continuous Populations, Regional Science and Urban Economics, 20, 473-508.

Bartle, R. G., 1976, The Elements of Real Analysis (2 ${ }^{\text {nd }}$ edition), New York, John Wiley \& Sons.

Bergstom, T. and R. C. Cornes, 1983, Independence of Allocative Efficiency from Distribution in the Theory of Public Goods, Econometrica, 51, 1753-1765.

Berliant, M. and M. Fujita, 1992, Alonso's Discrete Population Model of Land Use: Efficient Allocations and Competitive Equilibria, International Economic Review, 33, 535-566.

Berliant, M., Y.Y. Papageorgiou and Ping Wang, 1990, On Welfare Theory and Urban Economics, Regional Science and Urban Economics, 20, 245-261.

Berliant, M. and T. ten Raa, 1991, On the Continuum Approach of Spatial and Some Local Public Goods or Product Differentiation Models: Some Problems, Journal of Economic Theory, 55, 95-120.

Berliant, M. and T. ten Raa, 1994, Regional science: The state of the art, Regional Science and Urban Economics, 23, 631-647.

Berliant, M. and Ping Wang, 1993, Endogenous Formation of a City without Agglomerative Externalities or Market Imperfections: Marketplaces in a Regional Economy, Regional Science and Urban Economics, 23, 121-144.

Brueckner, J.K., 1979, Equilibrium in a System of Communities with Local Public Goods, Economics Letters, 2, 387-393.

Ellickson, B., 1979, Competitive Equilibrium with Local Public Goods, Journal of Economic Theory, 21, 46-61.

Fujita, M., 1986, Optimal Location of Public Facilities: Area Dominance Approach, Regional Science and 
Urban Economics, 16, 241-268.

Hochman, O., 1982, Congestable Local Public Goods in an Urban Setting, Journal of Urban Economics, $11,290-310$.

Hochman, O. and D. Pines, 1997, On the Agglomeration of Non-Residential Activities in an Urban Area, Working Paper, Ben-Gurion University of the Negev, Israel, November 1997.

Klein, E., and A.C. Thompson, 1984, Theory of Correspondences: Including Applications to Mathematical Economics, Wiley, New York, NY.

Koopmans, T.C. and M.J. Beckmann, 1957, Assignment Problems and the Location of Economic Activities, Econometrica, 25, 53-76.

Lucas, R.E. Jr, 1990, Liquidity and Interest Rates, Journal of Economic Theory, 50, 237-64.

Peng, S.K., 1996, The Location of Government Facilities and Equilibrium Urban Configuration, The Annals of Regional Science, 30, 247-272.

ReVelle, C., 1987, Urban Public Facility Location, in: E.S. Mills, ed., Handbook of Urban Economics, North-Holland, Amsterdam, 1053-1096.

Sakashita, N., 1987, Optimal Location of Public Facilities Under Influence of the Land Market, Journal of Regional Science, 27, 1-12.

Scotchmer, S., 1986, Local Public Goods in an Equilibrium: How Pecuniary Externalities Matter. Regional Science and Urban Economics, 16, 463-481.

Starrett, D.A., 1991, Foundations of Public Economics, Cambridge, Cambridge University Press.

Thisse, J.-F. and D.E. Wildasin, 1992, Public Facility Location and Urban Spatial Structure: Equilibrium and Welfare Analysis, Journal of Public Economics, 48, 83-118.

Wang, P., 1990, Competitive Equilibrium Formation of Marketplaces with Heterogeneous Consumers, Regional Science and Urban Economics, 20, 295-304.

Wildasin, D.E., 1987, Theoretical Analysis of Local Public Economics, in: E.S. Mills, ed., Handbook of Urban Economics, North-Holland, Amsterdam, 429-476. 
Figure 1: Public Facility Configuration and Total Transportation Costs with $N=2$
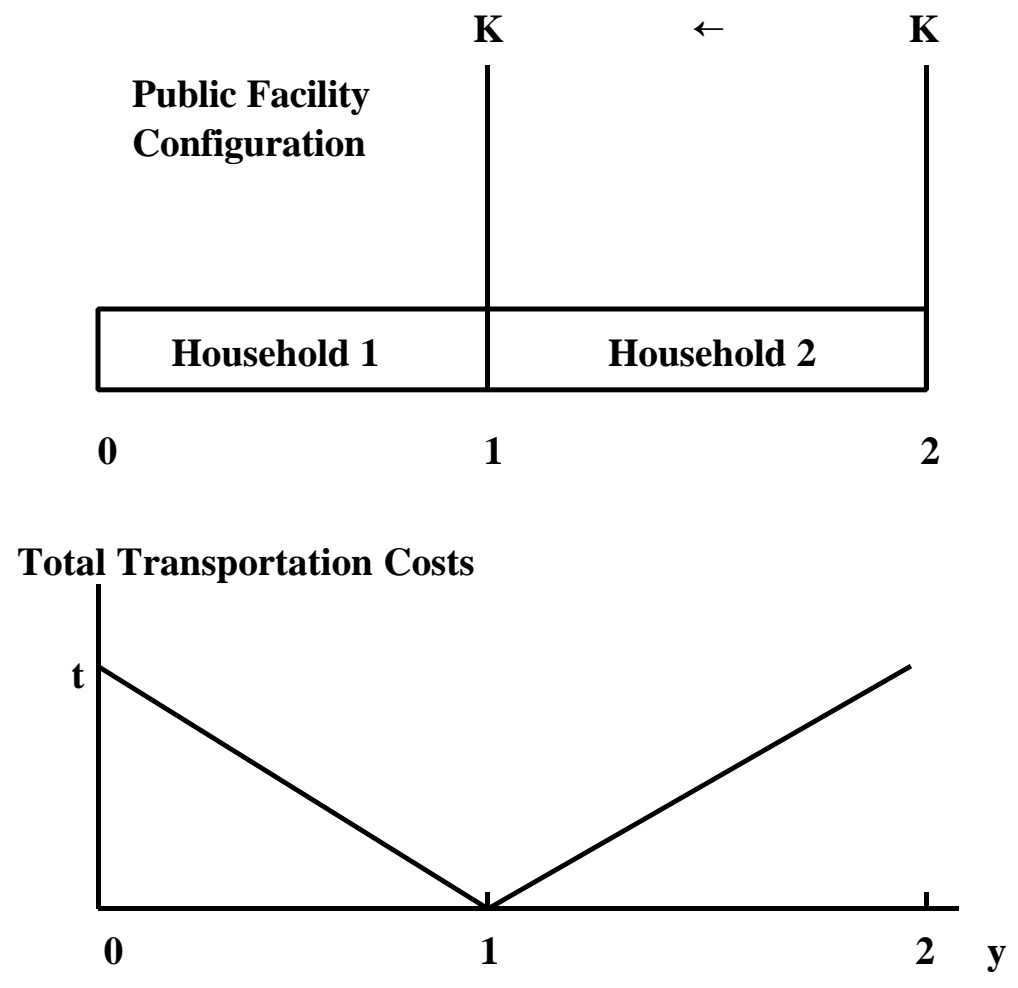
Figure 2: Public Facility Configuration and Total Transportation Costs with $N=3$

(a) The Case of Concentrated Configuration
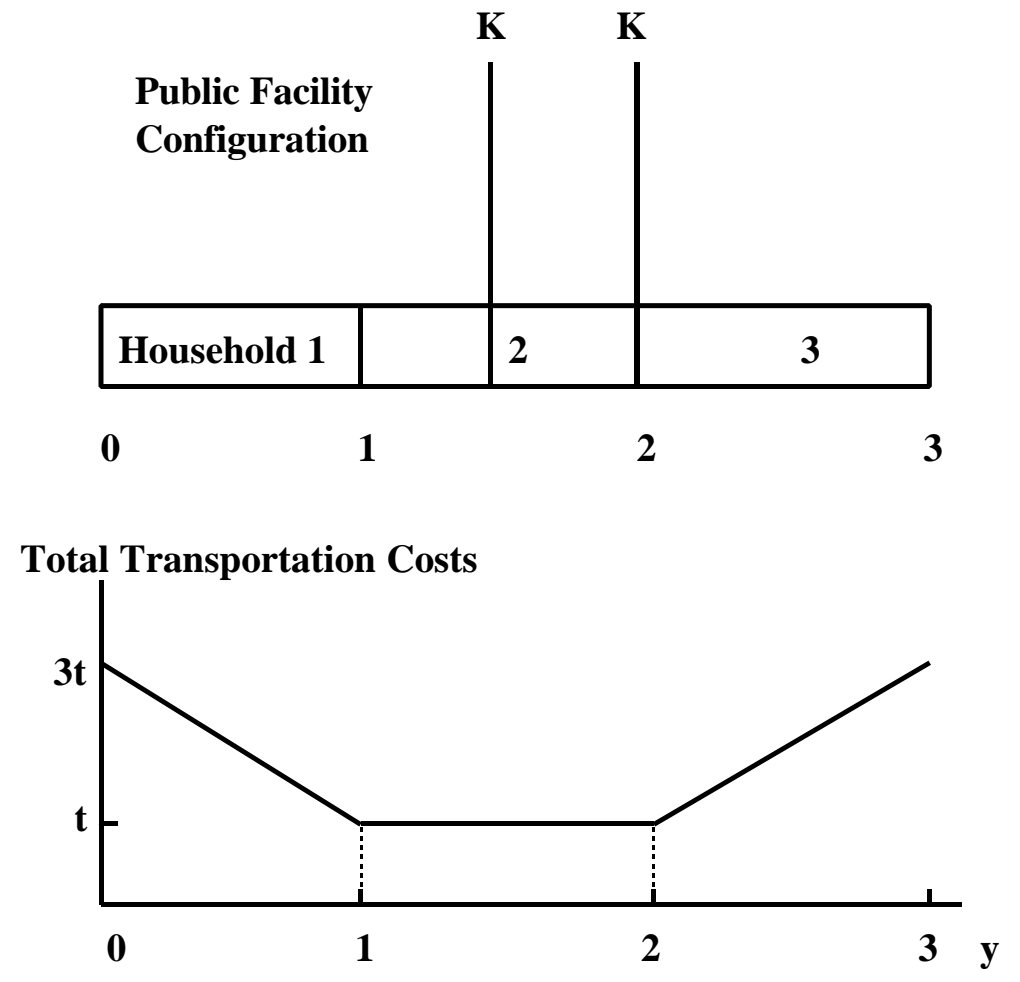

(b) The Case of Dispersed Configuration

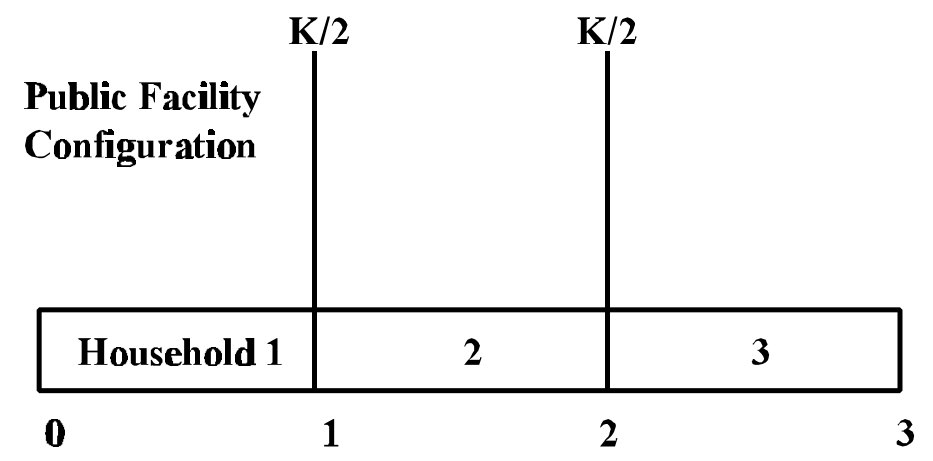

